\title{
The surface composition of Enceladus: clues from the Ultraviolet
}

\author{
Amanda R. Hendrix and Candice J. Hansen \\ Jet Propulsion Laboratory, California Institute of Technology, Pasadena, USA
}

\begin{abstract}
The reflectance of Saturn's moon Enceladus has been measured at far ultraviolet (FUV) wavelengths (115-190 nm) by Cassini's UltraViolet Imaging Spectrograph (UVIS). At visible and near infrared (VNIR) wavelengths Enceladus' reflectance spectrum is very bright, consistent with a surface composed primarily of $\mathrm{H}_{2} \mathrm{O}$ ice. At FUV wavelengths, however, Enceladus is surprisingly dark - darker than would be expected for pure water ice. We find that the low FUV reflectance of Enceladus can be explained by the presence of a small amount of $\mathrm{NH}_{3}$ and a small amount of a tholin in addition to $\mathrm{H}_{2} \mathrm{O}$ ice on the surface.
\end{abstract}

Keywords. planets and satellites: general; planets and satellites: Enceladus

\section{Introduction}

The canonical method for studying surface composition is the analysis of spectral reflectance measurements at visible-near infrared (VNIR) wavelengths. Using this method, the primary surface component of the Saturnian satellites has been found to be water ice (e.g., McCord et al. 1971; Fink et al. 1976; Morrison et al. 1976; Cruikshank 1980; Clark et al. 1984), possibly in mixtures with other constituents (e.g., Emery et al. 2005; Verbiscer et al. 2006; Clark et al. 2008). Most of the published spectral data of Enceladus focus on the near-IR: the Emery et al. (2005) and Cruikshank et al. (2005) spectra extend as short as $800 \mathrm{~nm}$, as do the Verbiscer et al. (2006) Enceladus spectra. Grundy et al. (1999) present Enceladus spectra at wavelengths as short as $1200 \mathrm{~nm}$. Published Enceladus spectra from the Cassini Visible-Infrared Mapping Spectrometer (VIMS) extend as short as $1000 \mathrm{~nm}$ (Brown et al. 2006). At visible wavelengths, the only published spectral information on Enceladus is photometric data from Voyager (Buratti \& Veverka 1984; Buratti 1984) and Hubble Space Telescope (HST) (Verbiscer et al. 2005). Buratti (1984) pointed out that, at visible wavelengths, the brighter Saturnian satellites are less spectrally red, suggesting that the visibly darker satellites contain more contaminants that are reddish; in particular, Buratti et al. (1990) note that Enceladus' "high albedo and flat spectrum between 350 and $590 \mathrm{~nm}$ imply that its surface layer is depleted in opaque materials." The visible spectrum $(350-600 \mathrm{~nm})$ of Enceladus is flat to bluish, consistent with an almost pure water ice surface (Buratti 1984; Buratti \& Veverka 1984). Toward shorter UV wavelengths, the spectrum of Enceladus has not been previously studied. Here we investigate the reflectance spectrum of Enceladus as measured by the Cassini Ultraviolet Imaging Spectrograph (UVIS) (Esposito et al. 2004).

The Enceladus observation discussed here was obtained on 27 May 2007, at an altitude of approximately $620,000 \mathrm{~km}$. The observation lasted 70 minutes, during which the solar phase angle decreased from $3.1^{\circ}$ to $1.0^{\circ}$ and increased back to $3.1^{\circ}$. The average phase angle during the observation was $1.93^{\circ}$. The sub-spacecraft latitude was $13^{\circ} \mathrm{S}$ during the observation; the sub-spacecraft longitude transitioned from $26^{\circ} \mathrm{W}$ to $33^{\circ} \mathrm{W}$. The size 
of the Enceladus disk during this observation was $0.8 \mathrm{mrad}$; the size of a UVIS pixel during this observation was $1.0 \times 1.5 \mathrm{mrad}$, so Enceladus was sub-pixel in size and UVIS measured a disk-average spectrum.

We calculate the disk-average reflectance by taking into account the size of the body in the UVIS slit and dividing by the solar spectrum as measured by SORCE SOLSTICE (McClintock et al. 2000), scaled to 9.2 AU, Enceladus' heliocentric distance on the day of the observation. We use the Hapke (2002) model to represent the reflectance spectra of individual species given their optical constants, and we use spectral mixing models with varying amounts of different species to try to replicate the spectral magnitude and shape observed at Enceladus, following the methods outlined by Hendrix \& Hansen (2008).

Laboratory measurements of water ice in the ultraviolet have had varying results. Several measurements of the optical constants $n$ and $k$ have been made: Warren (1984) provides a compilation of the measurements of hexagonal ice in the 44-2270 nm wavelength range and Warren \& Brandt (2008) recently updated the compilation. Absorption by ice in the near- and mid-UV $(\sim 200-400 \mathrm{~nm})$ is very weak (Warren \& Brandt 2008) but the location of minimum absorption is unclear. There is a lack of optical constant information in the ultraviolet, and as a result, the Warren (1984) and Warren \& Brandt (2008) compilations include an interpolation between $161 \mathrm{~nm}$ and $180 \mathrm{~nm}$, the Warren (1984) compilation also interpolates between $185 \mathrm{~nm}$ and $400 \mathrm{~nm}$ (Fig. 3), and the Warren \& Brandt (2008) compilation extrapolates between 180 and $200 \mathrm{~nm}$. The Warren \& Brandt (2008) compilation uses a different data set in the 390-600 $\mathrm{nm}$ range than the Warren (1984) compilation, and leaves a gap in the 200-390 $\mathrm{nm}$ range. The Warren \& Brandt (2008) data focus mainly on temperatures applicable at Earth; thus, these data are not appropriate for Enceladus-like (e.g., $80 \mathrm{~K}$ ) temperatures. Nevertheless, we use the Warren \& Brandt (2008)) optical constants for water ice in our models, as no other data are currently available. (As pointed out by Warren (1984), Shibaguchi et al. (1977) measured only small variations in optical density with temperature $(83 \mathrm{~K}-160 \mathrm{~K})$ in the 120-150 nm region; any temperature dependence in the optical constants of water ice at $\lambda>150 \mathrm{~nm}$ is unknown.)

\section{The far-UV spectrum of Enceladus does not agree with pure $\mathrm{H}_{2} \mathrm{O}$ ice}

In Fig. 1a is shown the far-UV (FUV) reflectance spectrum of Enceladus. The UVIS spectrum is dark at wavelengths $<165 \mathrm{~nm}$; the onset in brightness due to water ice is detected at $165 \mathrm{~nm}$; at wavelengths $\sim 170-\sim 185 \mathrm{~nm}$, the spectrum is relatively flat, producing a "ledge" in the overall spectrum; the spectrum appears to begin increasing again (possibly the shoulder of an absorption edge) at $\sim 185 \mathrm{~nm}$. We combine the FUV spectrum with published VNIR data to create a composite spectrum (Fig. 1b). Clearly, Enceladus exhibits a strong, abrupt decrease in reflectance in the middle-UV (MUV) region, as suggested by the single MUV data point of Verbiscer et al. (2006), and because the FUV spectrum is much darker than the visible spectrum. Water ice exhibits a strong dropoff in reflectance in the UV - but the $\mathrm{H}_{2} \mathrm{O}$ absorption edge occurs in the FUV (at $\sim 165 \mathrm{~nm}$, depending on grain size as shown in Fig. 1a), and is not responsible for the decrease in brightness of Enceladus at $\lambda<336 \mathrm{~nm}$, nor for the darkness of the Enceladus spectrum at $\lambda=170-185 \mathrm{~nm}$. Thus, pure water ice models (Fig. 1) are much too bright to fit the UVIS Enceladus spectrum. 

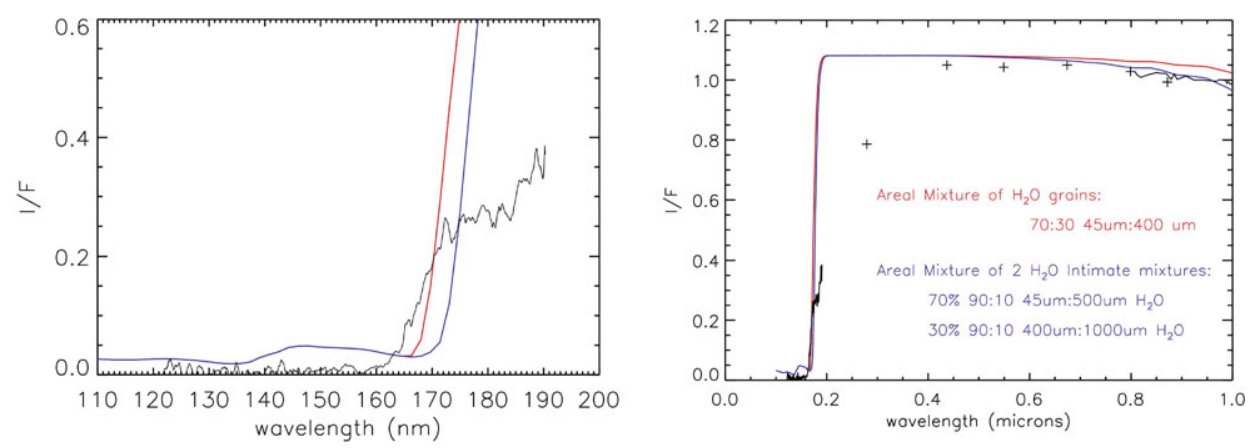

Figure 1. Enceladus spectra compared with water ice spectral models. (left) UVIS Enceladus disk-average reflectance spectrum at solar phase $=2^{\circ}$. (right) Enceladus data from UVIS and Verbiscer et al. (2005) (plus signs), also at $2^{\circ}$ solar phase angle. (red) areal mixture of 2 grain sizes; (blue) areal mixture of 2 intimate mixtures of 2 grain sizes. Both models are too bright to fit the UVIS data in the 160-190 $\mathrm{nm}$ range and do not exhibit the dropoff in brightness that evidently occurs in the near-UV $(\sim 300 \mathrm{~nm})$.

\section{Spectral mixture models}

We have investigated candidate species that could be present at Enceladus and could meet the criteria of being bright and featureless in the visible, largely featureless in the near-IR, featureless in the 120-190 nm region, and having a UV absorption edge in the 190-280 nm, with perhaps a weaker absorption in the 280-400 nm region. We tried different mixtures of $\mathrm{H}_{2} \mathrm{O}, \mathrm{CO}_{2}$, tholins and $\mathrm{NH}_{3}$. Other candidate species are naturally possible, but published optical constant data in the far-UV are not available. The UV spectrum of carbon dioxide frost is not consistent with the Enceladus spectrum, so we do not consider $\mathrm{CO}_{2}$ to be important in our considerations. Understanding that including more than $\sim 2 \%$ of either a tholin or $\mathrm{NH}_{3}$ adversely affects the spectral fits in the visible (as well as in the near-IR), we constrained the amount of those species to $1 \%$ in intimate mixtures with $\mathrm{H}_{2} \mathrm{O}$ ice. We find that mixture models with $1 \%$ each of ice tholin and $\mathrm{NH}_{3}$ result in spectra that are consistent with the Enceladus spectrum: they do not match exactly, but they reproduce the overall spectral features. We also find that if we model the $\mathrm{NH}_{3}-\mathrm{H}_{2} \mathrm{O}$ intimate mixture as being present on only a portion of the body (e.g., the south polar region), with pure water ice everywhere else on the surface, that we get poor fits to the Enceladus spectrum; areal mixtures with more than $\sim 20 \%$ pure $\mathrm{H}_{2} \mathrm{O}$ ice are simply too bright to agree with the UVIS spectrum; suggesting a more global coverage
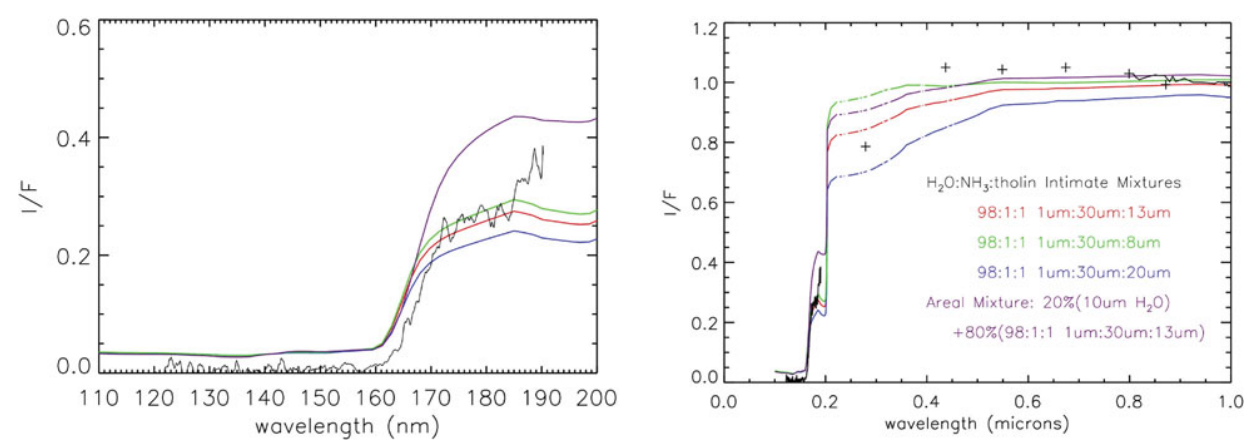

Figure 2. Enceladus data (as in Fig. 1) compared with spectral intimate mixture models using $\mathrm{H}_{2} \mathrm{O}$ ice, ice tholin and $\mathrm{NH}_{3}$ with varying grain sizes. 
by the intimate mixture, possibly due to E-ring grain interaction. Satisfactory fits are obtained when assuming global coverage of the $\mathrm{NH}_{3}-\mathrm{H}_{2} \mathrm{O}$-tholin mixture.

\section{Implications}

It is now known that Enceladus hosts water vapor jets with sources at the south polar hot spot (Hansen et al. 2006; Porco et al. 2006; Spencer et al. 2006; Dougherty et al. 2006). The plume gases include $>90 \% \mathrm{H}_{2} \mathrm{O}$ (Hansen et al. 2006; Waite et al. 2006) in addition to small amounts of $\mathrm{NH}_{3}(0.8 \%)$ and other trace species (Waite et al. 2006; Waite et al. 2009), including several types of hydrocarbons. UVIS likely detects the plume $\mathrm{NH}_{3}$ that has been redeposited on the surface on Enceladus; however, it appears that the $\mathrm{NH}_{3}$ is not present only in the south polar region, but globally, suggesting a different transport process such as E-ring interactions.

Though ammonia is not expected to be stable to photoloysis and radiolysis at the surface of Enceladus, ammonia hydrate may be stable, and we cannot rule out that it is not pure ammonia but ammonia hydrate (or a related species) that causes the strong UV dropoff. The presence of vaporous $\mathrm{NH}_{3}$ in the Enceladus plume (Waite et al. 2009) suggests that the $\mathrm{NH}_{3}$ could condense along with $\mathrm{H}_{2} \mathrm{O}$ in the E-ring), which would replenish $\mathrm{NH}_{3}$ on the surface of Enceladus via E-ring grain coating (Buratti 1988; Buratti et al. 1990), even in regions away from the south polar region. Redeposited plume material, along with coating by E-ring grains, contributes to the brightness of Enceladus' surface. Verbiscer et al. (2007) suggest that Enceladus is so bright because of this recoating, an idea first suggested by Buratti (1988) and supported by Hamilton \& Burns (1994).

\section{Acknowledgements}

This research was carried out at the Jet Propulsion Laboratory, California Institute of Technology, under a contract with the National Aeronautics and Space Administration.

\section{References}

Brown, R. H. et al. 2006, Science, 311, 1425

Buratti, B. 1984, Icarus, 59, 392

Buratti, B. J., Mosher, J. A., \& Johnson, T. V. 1990, Icarus, 87, 339

Buratti, B. J. \& Veverka, J. 1984, Icarus, 58, 254

Clark, R. N., Brown, R. H., Owensby, P. D., \& Steele, A. 1984, Icarus, 58, 265

Clark, R. N., Curchin, J. M., Jaumann, R., Cruikshank, D. P., Brown, R. H., Hoefen, T. M., Stephan, K., Moore, J. M., Buratti, B. J., Baines, K. H., Nicholson, P. D., \& Nelson, R. M. 2008, Icarus, 193, 372

Cruikshank, D. P. 1980, Icarus, 41, 246

Cruikshank, D. P., Owen, T. C., Dalle Ore, C., Geballe, T. R., Roush, T. L., de Bergh, C. Sandford, S. A., Poulet, F., Benedix, G. K., \& Emery, J. P. 2005, Icarus, 175, 268

Dougherty, M. K., Khurana, K. K., Neubauer, F. M., Russell, C. T., Saur, J., Leisner, J. S., \& Burton, M. E. 2006, Science, 311, 1406

Emery, J. P., Burr, D. M., Cruikshank, D. P., Brown, R. H., \& Dalton, J. B. 2005, A $\& A, 435$, 353

Esposito, L. W. et al. 2004. Space Sci. Rev., 115, 299

Fink, U., Larson, H. P., Gautier III., T. N., \& Treffers, R. R. 1976, ApJ, 207, L63

Grundy, W. M., Buie, M. W., Stansberry, J. A., \& Spencer, J. R. 1999, Icarus, 142, 536

Hamilton, D. P. \& Burns, J. A. 1994, Science, 264, 550 
Hansen, C. J., Esposito, L., Stewart, A. I. F., Colwell, J., Hendrix, A., Pryor, W., Shemansky, D., \& West, R. 2006, Science, 311, 1422

Hapke, B. W. 2002, Icarus 157, 523

Hendrix, A. R. \& Hansen, C. J. 2008, Icarus, 193, 323

McClintock, W. E., Rottman, G. J., \& Woods, T. N. 2000, Earth Observing System V, Proceedings of the SPIE, 4135, 225

McCord, T. B., Johnson, T. V., \& Elias, J. H. 1971, ApJ, 165, 413

Morrison, D., Cruikshank, D. P., Pilcher, C. B., \& Rieke, G. H. 1976, ApJ, 207, L213

Porco, C. C. et al. 2006, Science, 311, 1393

Shibaguchi, T., Onuki, H., \& Onaka, R. 1977, J. Phys. Soc. Jpn., 42, 152

Spencer, J. R., Pearl, J. C., Segura, M., Flasar, F. M., Mamoutkine, A., Romani, P., Buratti, B. J., Hendrix, A. R., Spilker, L. J., \& Lopes, R. M. C. 2006, Science, 311, 1401

Verbiscer, A. J., French, R. G., \& McGhee, C. A. 2005, Icarus, 173, 66

Verbiscer, A. J., Peterson, D. E., Skrutskie, M. F., Cushing, M., Helfenstein, P., Nelson, M. J., Smith, J. D., \& Wilson, J. C. 2006, Icarus, 182, 211

Verbiscer, A., French, R., Showalter, M., \& Helfenstein, P. 2007, Science, 315, 815

Waite, J. H., Combi, M. R., Ip, W.-H., Cravens, T. E., McNutt Jr., R. L., Kasprzak, W., Yelle, R., Luhmann, J., Niemann, H., Gell, D., Magee, B., Fletcher, G., Lunine, J., \& Tseng W.-L. 2006, Science, 311, 1419

Waite, Jr., J. H., Lewis, W. S., Magee, B. A., Lunine, J. I., McKinnon, W. B., Glein, C. R., Mousis, O., Young, D. T., Brockwell, T., Westlake, J., Nguyen, M.-J., Teolis, B. D., Niemann, H. B., McNutt Jr., R. L., Perry, M., \& Ip, W.-H. 2009, Nature, 460, 487

Warren, S. G. 1984, Appl. Optics, 23, 1206

Warren, S. G. \& Brandt, R. E. 2008, J. Geophys. Res., 113, D14220, doi: 10.1029/2007JD009744 